UvA-DARE (Digital Academic Repository)

\title{
Discovery of a magnetar associated with the soft gamma repeater SGR 1900+14
}

Kouveliotou, C.; Strohmayer, T.E.; Hurley, K.; van Paradijs, J.A.; Finger, M.H.; Dieters, S.; Woods, P.; Thompson, C.; Duncan, R.C.

Published in:

Astrophysical Journal

DOI:

$10.1086 / 311813$

Link to publication

Citation for published version (APA):

Kouveliotou, C., Strohmayer, T. E., Hurley, K., van Paradijs, J. A., Finger, M. H., Dieters, S., Woods, P.,

Thompson, C., \& Duncan, R. C. (1999). Discovery of a magnetar associated with the soft gamma repeater SGR 1900+14. Astrophysical Journal, 510, L115-L118. https://doi.org/10.1086/311813

\section{General rights}

It is not permitted to download or to forward/distribute the text or part of it without the consent of the author(s) and/or copyright holder(s), other than for strictly personal, individual use, unless the work is under an open content license (like Creative Commons).

Disclaimer/Complaints regulations

If you believe that digital publication of certain material infringes any of your rights or (privacy) interests, please let the Library know, stating your reasons. In case of a legitimate complaint, the Library will make the material inaccessible and/or remove it from the website. Please Ask the Library: https://uba.uva.nl/en/contact, or a letter to: Library of the University of Amsterdam, Secretariat, Singel 425, 1012 WP Amsterdam, The Netherlands. You will be contacted as soon as possible. 


\title{
DISCOVERY OF A MAGNETAR ASSOCIATED WITH THE SOFT GAMMA REPEATER SGR $1900+14$

\author{
C. Kouveliotou, ${ }^{1,2}$ T. Strohmayer ${ }^{3}$ K. Hurley, ${ }^{4}$ J. van Paradijs, ${ }^{5,6}$ M. H. Finger, ${ }^{1,2}$ S. Dieters, ${ }^{5}$ \\ P. Woods, ${ }^{5}$ C. Thompson, ${ }^{7}$ And R. C. DunCAN ${ }^{8}$ \\ Received 1998 September 10; accepted 1998 October 26; published 1998 December 10
}

\begin{abstract}
The soft gamma repeater SGR 1900+14 became active again on 1998 June after a long period of quiescence; it remained at a low state of activity until 1998 August, when it emitted a series of extraordinarily intense outbursts. We have observed the source with the Rossi X-Ray Timing Explorer twice, during the onset of each active episode. We confirm the pulsations at the $5.16 \mathrm{~s}$ period reported earlier from SGR $1900+14$. Here we report the detection of a secular spin-down of the pulse period at an average rate of $1.1 \times 10^{-10} \mathrm{~s} \mathrm{~s}^{-1}$. In view of the strong similarities between SGRs, we attribute the spin-down of SGR 1900+14 to magnetic dipole radiation, possibly accelerated by a quiescent flux, as in the case of SGR 1806-20. This allows an estimate of the pulsar dipolar magnetic field, which is $(2-8) \times 10^{14} \mathrm{G}$. Our results confirm that SGRs are magnetars.
\end{abstract}

Subject headings: stars: neutron — stars: magnetic fields

\section{INTRODUCTION}

Soft gamma repeater sources (SGRs) were recognized as a new class of objects in the late 1980s (Atteia et al. 1987; Laros et al. 1987; Kouveliotou et al. 1987). To date, only four SGRs are confirmed; a fifth SGR source may have been detected twice (Hurley et al. 1997; Kouveliotou et al. 1997; Smith et al. 1997), but its existence is not yet firmly established. What distinguishes these transient soft $\gamma$-ray bursters from the "classic" $\gamma$-ray burst sources is their recurrence and the much softer spectra of their bursts. SGRs undergo random intervals of intense activity, during which they emit bunches of up to hundreds of very short, low-energy bursts. The bursts vary in duration and temporal structure from simple, single pulses lasting less than $10 \mathrm{~ms}$ (Kouveliotou 1995) to long, highly complex events that comprise over 40 very short subpulses, each lasting tens of milliseconds (Kouveliotou et al. 1998b; Hurley et al. 1999b). The SGR burst arrival times seem to be distributed lognormally, similar to the distribution of earthquakes (Cheng et al. 1996; Hurley et al. 1995).

Three SGRs have firm associations, based on positional coincidences, with supernova remnants (SNRs). The position of SGR $1900+14$ has only recently been determined with sufficient accuracy (Hurley et al. 1999b) to establish that it lies close to the SNR G42.8+0.6, whose association with SGR $1900+14$ was suggested earlier (Kouveliotou et al. 1994; Hurley et al. 1994; Vasisht et al. 1994). The SGR/SNR associations indicate that SGRs are neutron stars, a conjecture strongly supported by the detection of a 3 minute train of $8 \mathrm{~s}$ pulsations following the famous 1979 March 5 event from SGR 0526-66 (Mazets et al. 1979).

\footnotetext{
${ }^{1}$ Universities Space Research Association.

${ }^{2}$ NASA Marshall Space Flight Center, ES-84, Huntsville, AL 35812; chryssa.kouveliotou@msfc.nasa.gov.

${ }^{3}$ NASA Goddard Space Flight Center, Greenbelt, MD 20771.

${ }^{4}$ University of California at Berkeley, Space Sciences Laboratory, Berkeley, CA 94720-7450.

${ }^{5}$ Department of Physics, University of Alabama in Huntsville, Huntsville, AL 35899.

${ }^{6}$ Astronomical Institute "Anton Pannekoek," University of Amsterdam, Kruislaan 403, 1098 SJ Amsterdam, The Netherlands.

${ }^{7}$ Department of Physics and Astronomy, University of North Carolina, Phillips Hall, Chapel Hill, NC 27599-3255.

${ }^{8}$ Department of Astronomy, University of Texas, RLM 15.308, Austin, TX $78712-1083$.
}

Searches for pulsed emission in the SGR persistent flux were finally successful when observations of SGR 1806-20 with the Rossi X-Ray Timing Explorer (RXTE) in 1996 November revealed a $7.47 \mathrm{~s} X$-ray pulsar (Kouveliotou et al. 1998a). $R X T E$ observations of the newly discovered SGR 1621-47 (Kouveliotou et al. 1998c) indicated weak evidence of $6.7 \mathrm{~s}$ pulsations from the source (Dieters et al. 1998). Finally, in recent observations (1998 April) of SGR 1900+14, Hurley et al. (1999a) detected pulsed emission with a 5.16 s period. The pulsations of SGR 1806-20 show a secular spin-down. As argued by Kouveliotou et al. (1998a), this spin-down is due to magnetic dipole radiation; the corresponding dipolar component of the magnetic field of SGR $1806-20$ exceeds $10^{14} \mathrm{G}$, thus establishing SGRs as "magnetars" (Duncan \& Thompson 1992; Thompson \& Duncan 1995).

We report the results of observations with $R X T E$ that were obtained soon after the reactivation of the source was detected with the Burst and Transient Source Experiment (BATSE) on the Compton Gamma Ray Observatory and Ulysses in 1998 May (Hurley et al. 1998b; Kouveliotou et al. 1998b). Additional observations were obtained after the recent extraordinary source activity of 1998 August (Remillard, Smith, \& Levine 1998), when it emitted several events, one similar to the 1979 March 5 event (Cline, Mazets, \& Golenetskii 1998; Hurley et al. 1998a; Feroci et al. 1998). During these observations, we detected the $5.16 \mathrm{~s}$ pulse period (Hurley et al. 1998c, 1999a) and found that it shows a secular spin-down at a rate similar to that observed for SGR 1806-20 (Kouveliotou et al. 1998d). The great similarities between SGRs indicate that the causes of the spin-down of the pulsations in SGR 1900+14 and SGR 1806-20 are the same; i.e., SGR $1900+14$ is also a magnetar; its dipolar magnetic field strength is $(2-8) \times 10^{14} \mathrm{G}$.

\section{RXTE OBSERVATIONS}

\subsection{The 1998 May Observation}

We observed SGR 1900+14 between 1998 May 31 and June 9 for a total of $41.7 \mathrm{ks}$ on-source. ${ }^{9}$ After excluding all bursts from the time series, the data were energy-selected $(2-20 \mathrm{keV})$, barycentered, and binned at $0.25 \mathrm{~s}$ resolution. We calculated a

\footnotetext{
${ }^{9}$ The start and end times of the individual observations can be found at http://heasarc.gsfc.nasa.gov, which is maintained at NASA/GSFC by S. Calvo.
} 

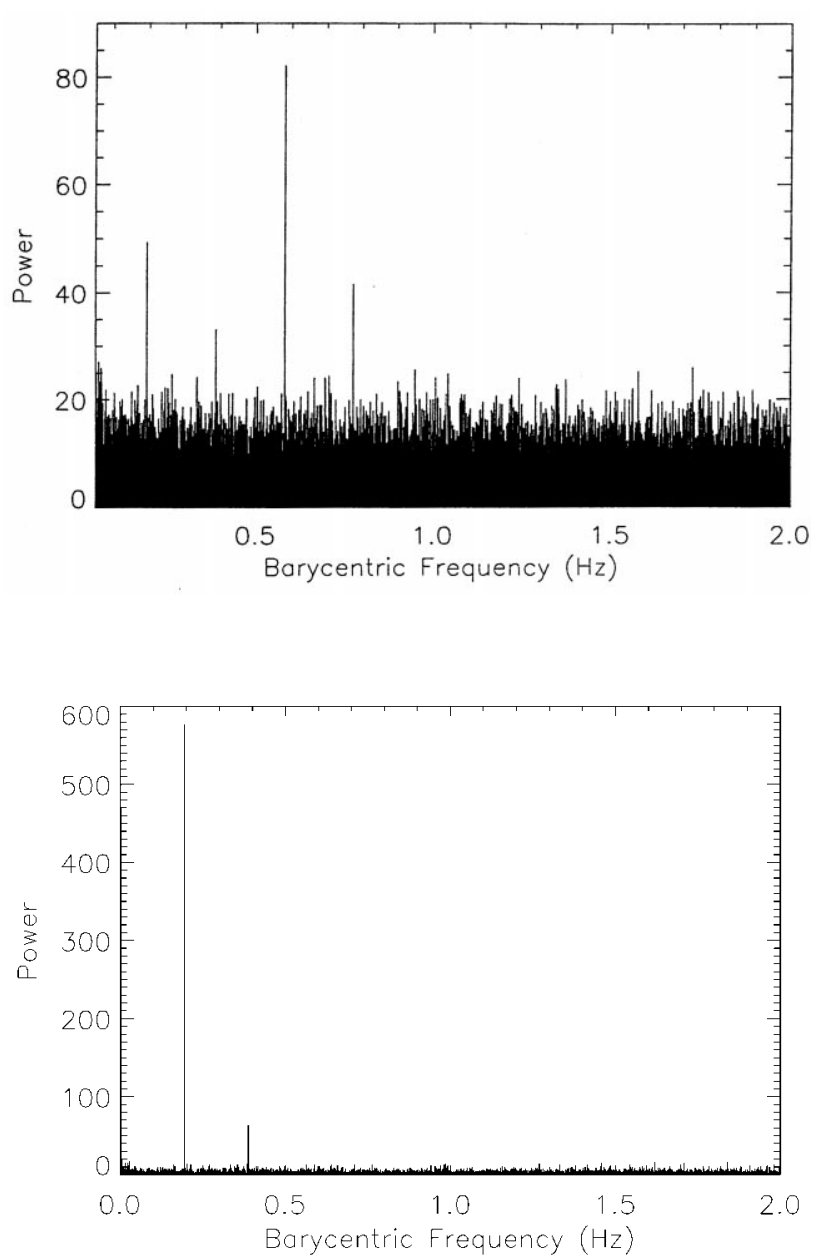

FIG. 1.-Top panel: PDS of the 1998 May RXTE observations of SGR $1900+14$. The highest peak in the spectrum corresponds to the fundamental period of $5.159142 \mathrm{~s}$; the three less intense peaks are the harmonics identified in the text. Bottom panel: PDS of the 1998 August 28 RXTE observation. The two highest peaks are the fundamental period at $5.160199 \mathrm{~s}$ and its second harmonic.

fast Fourier power spectrum of the resulting light curve, searching between 0 and $2.0 \mathrm{~Hz}$ for the $5.158975(7) \mathrm{s}$ period reported for SGR 1900+14 from the Advanced Satellite for Cosmology and Astrophysics (ASCA) data (Hurley et al. 1999a). The top panel of Figure 1 shows the power density spectrum (PDS) of the fast Fourier transform (FFT); we can clearly see the fundamental period at $5.159142(3)$ s $(0.193831 \mathrm{~Hz})$ (JD $2,450,970.5)$ and its first, second, and third harmonics. The chance probability of detecting this signal at the SGR $1900+14$ period is less than $3 \times 10^{-30}$. This number is derived after we averaged the power of the fundamental and the three harmonics between 0 and $1.0 \mathrm{~Hz}$, taking into account the number of trials. We do not find significant power at any other frequency; in particular, we do not detect a signal at the $89.17 \mathrm{~s}(0.011 \mathrm{~Hz})$ period from the X-ray pulsar XTE J1906+09 reported earlier (Marsden et al. 1998) in the error box of SGR 1900+14.

The top panel of Figure 2 shows the epoch-folded, Proportional Counter Array (PCA) pulse profile; it is very similar to the profile from the ASCA data obtained roughly a month earlier (Hurley et al. 1999a), although the energy ranges are not identical (the ASCA range is $2-10 \mathrm{keV}$ ). The two periods differ significantly; we estimate the period derivative between the
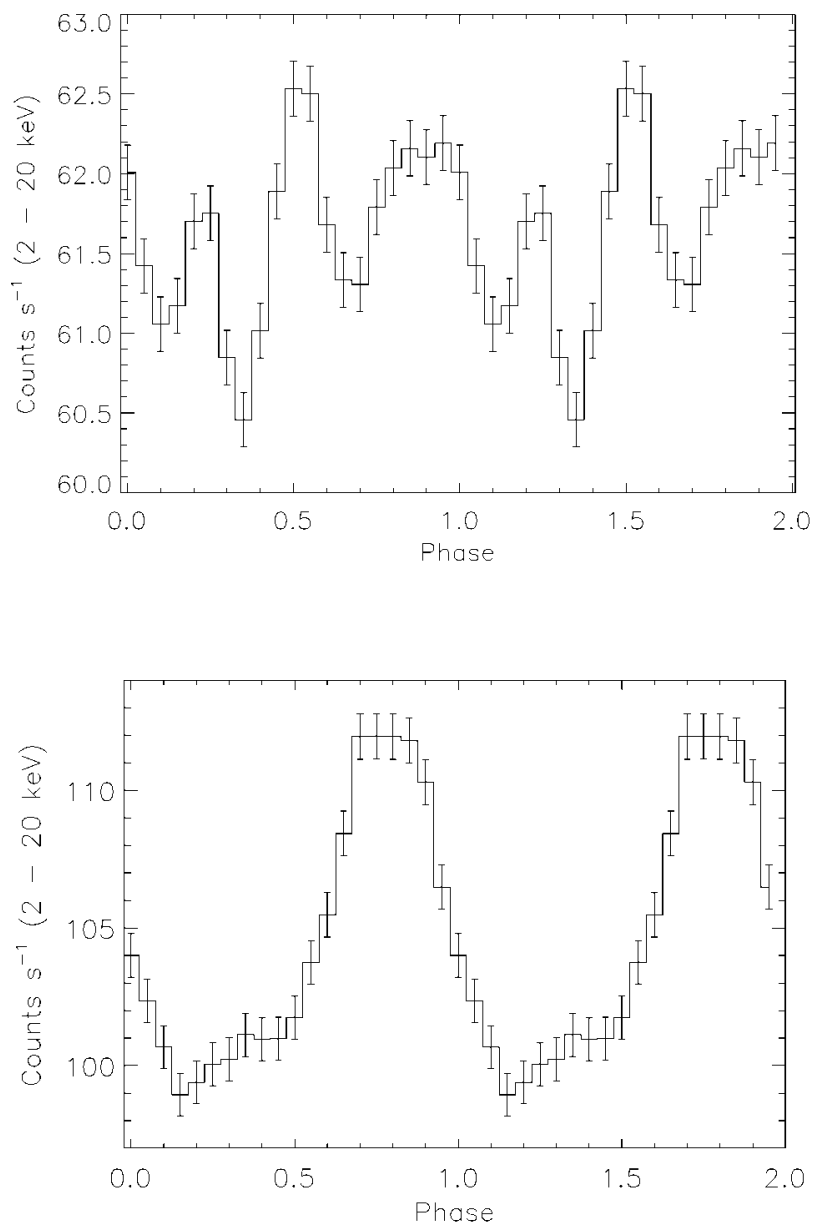

FIG. 2.-The epoch-folded pulse profile of SGR 1900+14 (2-20 keV) for (top) the 1998 May RXTE observations and (bottom) the 1998 August 28 $R X T E$ observation. The plot is exhibiting two phase cycles.

ASCA and RXTE observations to be $\dot{P}=5.44(24) \times 10^{-11} \mathrm{~s}$ $\mathrm{s}^{-1}$. Using a pulse-folding analysis on the PCA data alone, in which the period is assumed to be given by $P=P_{0}+\dot{P} t$, we find that during the 10 days of the PCA observations, a significant change of the period occurred. The best fit is $\dot{P}=$ $(1.10 \pm 0.17) \times 10^{-10} \mathrm{~s} \mathrm{~s}^{-1}$. We discuss the difference between the two $\dot{P}$ values in $\S 3$.

\subsection{The 1998 August Observation}

SGR 1900+14 started triggering BATSE again on $1998 \mathrm{Au}-$ gust 28. Prior to this first trigger, the RXTE/All-Sky Monitor had detected a rising transient source from the same direction as SGR 1900+14 (Remillard et al. 1998). In addition, the Ulysses and KONUS-Wind spacecraft recorded an extremely intense event on 1998 August 27, which was very similar to the 1979 March 5 event (Cline et al. 1998; Hurley et al. 1998a); during this event, the source was occulted by the Earth for BATSE (Kouveliotou et al. 1998e). A new series of Target of Opportunity observations was initiated with RXTE (see footnote 9 ). We have analyzed $2.5 \mathrm{ks}$ of on-source data obtained on 1998 August 28. From a pulse-timing analysis, we find the pulsar period to be $P=5.160199$ (2) s (on JD 2,451,056.5); the $P$ during the 1998 August observation is 1.14(23) $\times$ $10^{-10} \mathrm{~s} \mathrm{~s}^{-1}$. The bottom panel of Figure 1 shows the PDS of the FFT; the fundamental and the first harmonic dominate this 
spectrum. The average $\dot{P}$ between the first and second $R X T E$ observations is $1.406(5) \times 10^{-10} \mathrm{~s} \mathrm{~s}^{-1}$, which is 2.6 times the rate between the ASCA and the first RXTE observation.

The folded pulse profiles obtained during 1998 May and August are clearly different (see the bottom panel of Fig. 2), although they cover the same energy range; the 1998 August profile is also different from that obtained with ASCA. This is reminiscent of SGR 1806-20 (Kouveliotou et al. 1998a), for which we observed a spiky pulse profile with substructure when the source was "inactive" (ASCA observation), whereas a smooth, almost sinusoidal profile appears after the source had become very active ( $R X T E$ observation). The variable pulse profile of SGR $1900+14$ could be ascribed to residual heat output in the active region following the August 27 event.

The evolution of $\dot{P}$ is shown in Figure 3, where we plot the rate of spin-down calculated between each consecutive observation as well as within each $R X T E$ observation. Note that the spin-down rate remains practically constant after 1998 June.

\subsection{Search for Orbital Period}

In the 1998 August $R X T E$ observations, we found systematic departures of pulse phases from the best-fit ephemeris. These departures of $\sim 0.05$ cycles typically persisted for a few thousand seconds. Since such signatures can potentially be due to binary orbital motion, we searched for periodic variations in the pulse phases.

We analyzed $53 \mathrm{ks}$ of PCA standard1 data from August 28 to September 2. After data from bursts were eliminated, the rates were divided into $400 \mathrm{~s}$ intervals, with a pulse profile determined for each interval. Pulse phases were then determined by correlation with the average pulse profile. A search for sinusoidal variations was conducted for orbital periods from $10^{3}$ to $10^{6} \mathrm{~s}$, but no orbital modulation was found. For periods in the range of $10^{3} \mathrm{~s}<P_{\text {orbit }}<7.5 \times 10^{4} \mathrm{~s}$, we can limit circular orbits to $a_{x} \sin i<0.2\left(P_{\text {orbit }} / 10^{4}\right)^{0.15} \mathrm{~s}$ (95\% confidence). For $8 \times 10^{3} \mathrm{~s}<P_{\text {orbit }}<8 \times 10^{5} \mathrm{~s}$, possible companion masses must be below $0.1 M_{\odot} / \sin i$, assuming a $1.4 M_{\odot}$ neutron star.

It is likely that the short-term departure of the pulse phases from the long-term ephemeris is due to pulse-shape variations. We see minor changes in the pulse shape over the course of the August observations, while more dramatic changes are evident between the first and second RXTE observations.

\subsection{Spectral Analysis}

We have performed spectral fits using XSPEC on the persistent emission data for both RXTE observations. In both spectra, we find evidence of a line at $\sim 6.7 \mathrm{keV}$; the best fit was obtained for a power-law + Gaussian model. The values for the power-law (photon) index, $N_{\mathrm{H}}$, and line centroid are (2.1, $\left.3.4 \times 10^{22} \mathrm{~cm}^{-2}, 6.6 \mathrm{keV}\right)$ and $\left(3.1,5.5 \times 10^{22} \mathrm{~cm}^{-2}, 6.5 \mathrm{keV}\right)$ for 1998 June 2 and August 28, respectively; the line is more significant in the first observation. The power-law index and $N_{\mathrm{H}}$ values for June 2 are consistent with the values reported from the ASCA data (Hurley et al. 1999a); the August 28 data exhibit a higher $N_{\mathrm{H}}$ and a softer spectrum. We cannot confidently attribute the line to SGR $1900+14$ since the $R X T E$ field of view is $\sim 10$ times larger than that of ASCA and may include contamination from other sources-such as XTE J1906+09, which was active at least after 1998 August 30 (Takeshima, Corbet, \& Swank 1998)—as well as from the diffuse Galactic emission.

We have further estimated the unabsorbed persistent flux

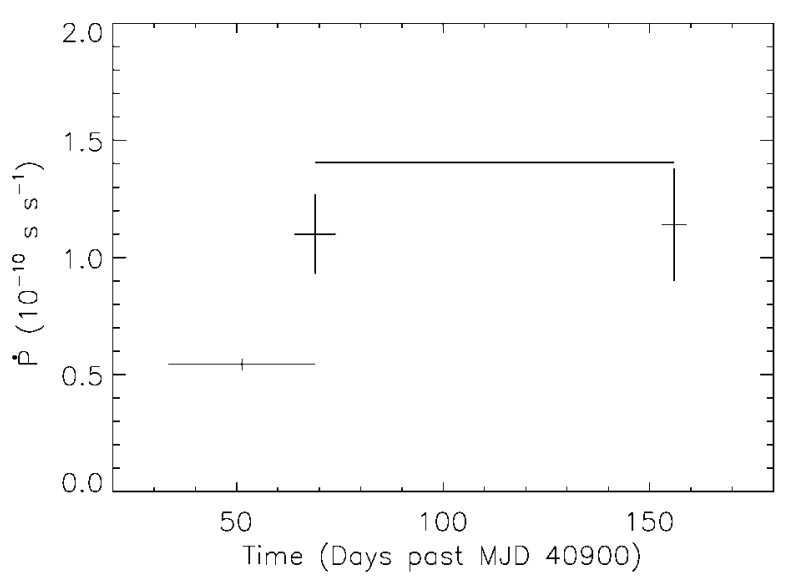

FIG. 3. - The evolution of $\dot{P}$ vs. time since the first period measurement of SGR $1900+14$ with ASCA (Hurley et al. 1999a). The time is given in Modified Julian Dates (MJDs).

between 2 and $10 \mathrm{keV}$ in order to compare it directly with the ASCA flux of $1.28 \times 10^{-11} \mathrm{ergs} \mathrm{cm}^{-2} \mathrm{~s}^{-1}$ that was reported for 1998 April 30 (Hurley et al. 1999a). For the 1998 June 2 and August 28 observations, the fluxes are $\sim 4.4 \times 10^{-11}$ and $\sim 9.9 \times 10^{-11} \mathrm{ergs} \mathrm{cm}^{-2} \mathrm{~s}^{-1}$, respectively (after taking into account the effect of the 0.441 offset of the RXTE pointing). Assuming a distance to the source of $\sim 7 \mathrm{kpc}$ (Hurley et al. 1994; Vasisht et al. 1994), we find that the luminosity of SGR $1900+14$ varied from $2.4 \times 10^{35}$ to $2.1 \times 10^{36} \mathrm{ergs} \mathrm{s}^{-1}$; i.e., it was a factor of $\sim 10$ higher during its second active episode. Although we cannot exclude the contribution of the diffuse Galactic emission and source contamination in the absolute values of these estimates, we believe that the ratio of the two values yields the true luminosity increase between the two episodes.

\section{DISCUSSION}

We have confirmed the second case of a pulsar (Hurley et al. 1999a) associated with the persistent emission of an SGR and have been able to measure a secular increase in its period. The first case, SGR 1806-20, had a very similar period and period derivative of $7.47 \mathrm{~s}$ and $8.3 \times 10^{-11} \mathrm{~s} \mathrm{~s}^{-1}$, respectively (Kouveliotou et al. 1998a). We have argued that the secular spin-down of SGR 1806-20 was due to torques from magnetic dipole radiation (MDR) and a relativistic wind, and we have estimated the pulsar characteristic spin-down age and (dipolar) magnetic field strength to be $\sim 1500 \mathrm{yr}$ and $8 \times 10^{14} \mathrm{G}$, respectively. Our earlier observations demonstrated the existence of magnetars-neutron stars with superstrong magnetic fields (Duncan \& Thompson 1992) — and supported models attributing SGR bursts to crustquakes produced by magnetic stress (Thompson \& Duncan 1995).

In view of the strong similarities between SGRs, it would appear that in SGR $1900+14$, the spin-down is also the result of a magnetized wind. The almost constant $\dot{P} \simeq 1.1 \times 10^{-10} \mathrm{~s}$ $\mathrm{s}^{-1}$ observed in SGR $1900+14$ between the 1998 May and August RXTE observations (see Fig. 3) implies $B_{\star}=8 \times$ $10^{14} \mathrm{G}$, if the vacuum magnetic torque were the only torque acting on the star. The age of SGR $1900+14$ would be $P / 2 \dot{P} \sim 700 \mathrm{yr}$, i.e., so short that one must give up any physical association between it and the nearby SNR G42.8+0.6. First, the remnant is much older, and, second, a tangential velocity 
of $5 \times 10^{4} D_{7} \mathrm{~km} \mathrm{~s}^{-1}$ would be required at a distance of $7 D_{7}$ kpc. The smaller $\dot{P}=5.5 \times 10^{-11} \mathrm{~s} \mathrm{~s}^{-1}$ between the ASCA and the RXTE 1998 May observations could be ascribed to a glitch of magnitude $\triangle P / P=-4 \times 10^{-5}$ during that interval. Large glitches in the range $|\Delta P / P|=10^{-5}-10^{-4}$ are indeed implied by an extrapolation of the glitching behavior of young pulsars to the much slower spins of the SGR and the "anomalous Xray pulsar" (AXP) sources (Thompson \& Duncan 1996), but it is surprising that SGR $1900+14$ should not also have been observed to glitch during the great burst of activity associated with the August 27 event.

The spin-down rate of a magnetar, however, may be grossly underestimated by the vacuum dipole formalism. The dipole luminosity $L_{\mathrm{MDR}}=1.0 \times 10^{34}\left(B_{\star} / 10 B_{\mathrm{QED}}\right)^{2}\left(R_{\star} / 10 \mathrm{~km}\right)^{6} \times$ $(P / 5.16 \mathrm{~s})^{-4} \mathrm{ergs} \mathrm{s}^{-1}$ is much smaller than the quiescent X-ray luminosity $\left(L_{\mathrm{X}} \sim 10^{35}-10^{36} \mathrm{ergs} \mathrm{s}^{-1}\right.$ for both the AXP and SGR sources) as well as the time-averaged burst luminosity $\left(\left\langle L_{\mathrm{X}}\right\rangle \sim 3 \times 10^{35} \mathrm{ergs} \mathrm{s}^{-1}\right.$ during an active phase of SGR 1806-20) (Kouveliotou et al. 1998a). In this context, the quiescent particle emission that is driven by internal seismic activity increases the spin-down luminosity to $I \omega \dot{\omega}=$ $L_{\mathrm{MDR}}\left(L_{\mathrm{particle}} / L_{\mathrm{MDR}}\right)^{1 / 2}$ (Thompson \& Blaes 1998), and the simple estimate of the magnetic field derived above from $P$ and $\dot{P}$ is an overestimate. This effect is especially important for SGR 1806-20, where $L_{\text {particle }} \sim 10^{37} \mathrm{ergs} \mathrm{s}^{-1}$ is inferred from observations of the surrounding radio halo (Vasisht, Frail, \& Kulkarni 1995) on both small and large angular scales (Thompson \& Duncan 1996); for that source, the required value of $B_{\star}$ is reduced to $2 \times 10^{14} \mathrm{G}$.

Transport of a magnetic field through the core and rigid crust of a neutron star leads to two basic modes of energy release (Thompson \& Duncan 1996): internal frictional heat, which is partially conducted to the surface, and frequent low-energy fractures that couple to magnetospheric Alfvén modes and thence to energetic particles. In this model, an SGR burst is triggered only if the Alfvén mode has a large enough amplitude to cascade to a high wavenumber before leaking out of the magnetosphere (Thompson \& Blaes 1998).
If SGR 1900+14 turns on as a quiescent particle source $\left(L_{\text {particle }}>L_{\mathrm{MDR}}\right)$ during brief periods of burst activity, then its true age exceeds our measured value of $P / 2 \dot{P}$ and a physical association with SNR G42.8+0.6 (estimated age $\sim 10^{4}$ yr; Vasisht et al. 1995) becomes possible. A magnetic field $B_{\star}=2 \times 10^{14} t_{4}^{-1 / 2} \mathrm{G}$ is required for dipole radiation alone to spin down the star to a $5.16 \mathrm{~s}$ period at an age of $t_{4} \times 10^{4} \mathrm{yr}$. Given this value of $B_{\star}$, the period derivative is raised above its long-term average to $\dot{P}=1.1 \times 10^{-10} \mathrm{~s} \mathrm{~s}^{-1}$ if $L_{\text {particle }}=$ $1.7 \times 10^{36}\left(\dot{P} / 10^{-10}\right)^{2} t_{4}$ ergs s${ }^{-1}$, well below the particle luminosity inferred for SGR 1806-20. The total energy injected in relativistic particles between the two RXTE observations is $\sim 2 \times 10^{43}$ ergs (not including any particles injected during the August 27 event). A comparison with the energy of the 1" synchrotron bubble around SGR 1806-20 (Vasisht et al. 1995) suggests that radio flux may be detectable (at the $0.1 \mathrm{mJy}$ level). At this more advanced age, the tangential velocity needed to propel SGR $1900+14$ out of SNR G42.8+0.6 is $3400 D_{7} t_{4}^{-1}$ $\mathrm{km} \mathrm{s}^{-1}$. Several mechanisms could impart unusually high velocities to magnetars at birth (Duncan \& Thompson 1992).

The time dependence of $\dot{P}$ and quiescent $L_{\mathrm{X}}$ in SGR $1900+14$ is mirrored in the behavior of the AXP sources. As is the case for SGR $1806-20$, the value of $P / \dot{P}$ for SGR $1900+14$ is smaller than any of the (five) values measured so $\mathrm{far}^{10}$ for AXPs, which have also been suggested to be magnetars (Duncan \& Thompson 1995; Thompson \& Duncan 1996). This supports the suggestion (Kouveliotou et al. 1998a) that AXPs are a later, less active, phase in the evolution of SGRs.

We acknowledge support from the following grants: NASA grants NAG5-2755, NAG5-3674, and NAG5-4878 (J. v. P.); NASA grants NAG5-32490 and NAG5-4799 (C. K.); NASA grant NAG5-3100 (C. T.); Texas Advanced Research Project grant ARP-028 (R. C. D.); NASA grant NAG5-4238 (M. H. F.); and NASA grant NAG5-4882 (K. H.)

\footnotetext{
${ }^{10}$ The spin-down behavior of $1 \mathrm{E} 2259+586$, the best-studied AXP, was discussed most recently in Baykal et al. (1998).
}

\section{REFERENCES}

Atteia, J.-L., et al. 1987, ApJ, 320, L105

Baykal, A., Swank, J. H., Strohmayer, T., \& Stark, M. J. 1998, A\&A, 336, 173

Cheng, B., Epstein, R. I., Guyer, R. A., \& Young, C. 1996, Nature, 382, 518

Cline, T. L., Mazets, E. P., \& Golenetskii, S. V. 1998, IAU Circ. 7002

Dieters, S., Woods, P., Kouveliotou, C., \& van Paradijs, J. 1998, IAU Circ. 6962

Duncan, R. C., \& Thompson, C. 1992, ApJ, 392, L9

1995, in AIP Conf. Proc. 366, High Velocity Neutron Stars and Gamma-Ray Bursts, ed. R. E. Rothschild \& R. E. Lingenfelter (Woodbury: AIP), 111

Feroci, M., Soffitta, P., Costa, E., Frontera, F., \& Tavani, M. 1998, IAU Circ. 7005

Hurley, K., Cline, T., Mazets, E., \& Golenetskii, S. 1998a, IAU Circ. 7004 Hurley, K., et al. 1997, IAU Circ. 6743

Hurley, K., Kouveliotou, C., Mazets, E., \& Cline, T. 1998b, IAU Circ. 6929

Hurley, K., et al. 1999a, ApJ, 510, L111

Hurley, K., Kouveliotou, C., Murakami, T., \& Strohmayer, T. 1998c, IAU Circ. 7001

Hurley, K., Kouveliotou, C., Woods, P., Cline, T., Butterworth, P., Mazets, E., Golenetskii, S., \& Frederics, D. 1999b, ApJ, 510, L107

Hurley, K., McBreen, B., Dealaney, M., \& Britton, A. 1995, Ap\&SS, 231, 81

Hurley, K., Sommer, M., Kouveliotou, C., Fishman, G., Meeghan, C., Cline,

T., Boer, M., \& Niel, M. 1994, ApJ, 431, L31

Kouveliotou, C. 1995, Ap\&SS, 231, 49
Kouveliotou, C., et al. 1987, ApJ, 332, L21

- 1994, Nature, 368, 125

Kouveliotou, C., Fishman, G. J., Meegan, C. A., \& Woods, P. 1997, IAU Circ. 6743

Kouveliotou, C., et al. 1998a, Nature, 393, 235

Kouveliotou, C., Woods, P., Kippen, M., Briggs, M., \& Hurley, K. 1998b, IAU Circ. 6929

Kouveliotou, C., et al. 1998c, IAU Circ. 6944

Kouveliotou, C., Strohmayer, T., Hurley, K., van Paradijs, J., \& Woods, P. 1998d, IAU Circ. 7001

Kouveliotou, C., Strohmayer, T., Takeshima, T., Swank, J. H., \& Woods, P. 1998e, IAU Circ. 7003

Laros, J., et al. 1987, ApJ, 320, L111

Marsden, D., Gruber, D. E., Heindl, W. A., Pelling, M. R., \& Rothschild, R. E. 1998, ApJ, 502, L129

Mazets, E. P., et al. 1979, Nature, 282, 587

Remillard, R., Smith, D. A., \& Levine, A. 1998, IAU Circ. 7002

Smith, D. A., Levine, A. M., Morgan, E. H., Remillard, R. A., \& Rutledge, R. 1997, IAU Circ. 6743

Takeshima, T., Corbet, R. H. D., \& Swank, J. H. 1998, IAU Circ. 7008

Thompson, C., \& Blaes, O. 1998, Phys. Rev. D, 57, 3219

Thompson, C., \& Duncan, R. C. 1995, MNRAS, 275, 255 . 1996, ApJ, 473, 322

Vasisht, G., Frail, D. A., \& Kulkarni, S. R. 1995, ApJ, 440, L65

Vasisht, G., Kulkarni, S. R., Frail, D. A., \& Greiner, J. 1994, ApJ, 431, L35 